\title{
Effect of many-body interactions on the solid-liquid phase-behavior of charge-stabilized colloidal suspensions
}

\author{
J. Dobnikar ${ }^{a}$, R. Rzehak ${ }^{b}$ and H. H. von Grünberg ${ }^{a}$ \\ ${ }^{a}$ Universität Konstanz, Fachbereich Physik, D-78457 Konstanz, Germany \\ ${ }^{b}$ IFF, Forschungszentrum Jülich, D-52425 Jülich, Germany
}

\begin{abstract}
The solid-liquid phase-diagram of charge-stabilized colloidal suspensions is calculated using a technique that combines a continuous Poisson-Boltzmann description for the microscopic electrolyte ions with a molecular-dynamics simulation for the macroionic colloidal spheres. While correlations between the microions are neglected in this approach, many-body interactions between the colloids are fully included. The solid-liquid transition is determined at a high colloid volume fraction where many-body interactions are expected to be strong. With a view to the Derjaguin-Landau-Verwey-Overbeek theory predicting that colloids interact via Yukawa pair-potentials, we compare our results with the phase diagram of a simple Yukawa liquid. Good agreement is found at high salt conditions, while at low ionic strength considerable deviations are observed. By calculating effective colloid-colloid pair-interactions it is demonstrated that these differences are due to many-body interactions. We suggest a density-dependent pair-potential in the form of a truncated Yukawa potential, and show that it offers a considerably improved description of the solid-liquid phase-behavior of concentrated colloidal suspensions.
\end{abstract}

The classic Derjaguin-Landau-Verwey-Overbeek (DLVO) theory [1] predicts that an isolated pair of charged colloidal spheres in an aqueous salt solution interacts via a repulsive Yukawa potential at large separations. Direct experimental measurements have confirmed the general validity of this prediction [2, 3, 䑲. Though originally being designed only for pairs of colloids in isolation, it is common practice nowadays to apply the DLVO theory also to the much more complex case of colloids in concentrated suspensions, the situation which occurs in most technical applications. As we show in this letter, treating a colloidal suspension as a system of pairwise interacting Yukawa particles is valid only in a limited parameter regime. To this end a full Poisson-Boltzmann (PB) mean-field description of the colloidal interactions has been combined with a molecular-dynamics (MD) simulation, similar to Ref. [8], to calculate the solid-liquid phase-behavior of charge-stabilized colloidal suspensions. While correlations between the microions are neglected in this approach, many-body interactions between the colloids, mediated by the screening ionic fluid between and around the colloids, are fully included. By calculating the effective DLVO parameters for the same system, it is seen that at large volume fraction of colloids and low salt concentration, the description in terms of pair-wise interacting Yukawa particles fails dramatically.

\footnotetext{
${ }^{1}$ Jure.Dobnikar@uni-konstanz.de
} 
An estimate whether the DLVO pair potential is a good approximation in concentrated colloidal suspensions can be based on the ratio between i) the mean colloid-colloid distance, $d_{\mathrm{m}}=\rho^{-1 / 3}$, in suspensions with a density of colloidal particles $\rho$ and ii) the inverse Debye screening length $\kappa^{-1}$, which measures the thickness of the spherical double-layer around a single isolated colloidal sphere due to screening by the microions. If two or more such spherical double-layers overlap, the screening of the colloidal charges becomes incomplete and the charge distributions on the colloids involved begin to interact. If the colloid density is low, i.e. if $d_{\mathrm{m}} \kappa \gg 1$, it is obvious that such an overlap will almost always occur for pairs of colloids only. At high colloid densities, however, i.e. if $d_{\mathrm{m}} \kappa \sim 1$, there is a high probability that more than one other colloid is within the range of the double-layer $\kappa^{-1}$ around any colloidal particle. In this case many-body forces between the colloids become important, and the picture of pair-wise interacting particles ceases to be valid.

This scenario has been qualitatively confirmed by recent measurements of the effective colloidal pair-interaction potential as a function of the colloid density in $2 \mathrm{D}$ colloidal systems under low-salt conditions [5, 6]. In this context, many-body interactions show up as a densitydependence of the effective pair-potential [7]. Indeed, at low density, the measured pair-potential was Yukawa-like as predicted by DLVO theory, but at high density it showed clear deviations from a Yukawa-like behavior, particularly for inter-colloidal distances $r \gtrsim d_{\mathrm{m}}$ where the interaction decayed to zero much more rapidly than predicted by DLVO theory. This observed density-dependent "cut-off" of the interaction potential at the mean colloid-colloid distance $d_{\mathrm{m}}$ is a direct manifestation of many-body interactions in the system [5]. A simple physical explanation for this "cut-off" is based on the mechanism of macro-ion shielding [6]: the interaction between two colloids separated by a distance $r>d_{\mathrm{m}}$ is likely to be screened by a third colloid located somewhere between them. This screening effect of the macroionic charges, which is neglected in the DLVO theory, qualitatively explains the observed deviations from the Yukawa-potential. Motivated by these experiments, we here address the question to what extent many-body interactions affect macroscopic properties of $3 \mathrm{D}$ colloidal suspensions, specifically, the solid-liquid phase behavior.

Colloidal crystals near melting are simulated using a technique suggested by Fushiki [8] which combines a PB field description for the microions with a MD simulation for the macroionic colloids [9]. Specifically, we consider a fluid of highly charged identical colloidal spheres suspended in a structureless medium of dielectric constant $\varepsilon$ at temperature $T$. The Bjerrum length characterizing the medium is defined as $\lambda_{B}=e^{2} \beta / \varepsilon$, with $\beta=1 / k T, k$ Boltzmann's constant, and $e$ the elementary charge. The colloidal spheres have radius $a$, charge $-Z e$, and thus a surface charge density $-e \sigma=-Z e / 4 \pi a^{2}$. They are placed in a cubic box with periodic boundaries and the positions of their centers are denoted by $\mathbf{R}_{i}(i=1 \ldots N)$. The suspension is assumed to be in osmotic equilibrium with an electroneutral reservoir of monovalent point-like salt ions with total particle density $2 c_{s}$ and inverse Debye screening length $\kappa=\left(8 \pi \lambda_{B} c_{s}\right)^{1 / 2}$, and in thermal equilibrium with a heat bath at constant temperature. The density distribution of the electrolyte ions in the region $G$ exterior to the colloidal spheres is obtained from the normalized 
electrostatic potential $\phi(\mathbf{r})$ satisfying the PB equation

$$
\begin{aligned}
\nabla^{2} \phi(\mathbf{r}) & =\kappa^{2} \sinh \phi(\mathbf{r}), & & \mathbf{r} \in G \\
\mathbf{n}_{i} \nabla \phi & =4 \pi \lambda_{B} \sigma & & \mathbf{r} \in \partial G_{i}, i=1, \ldots, N,
\end{aligned}
$$

where $\partial G_{i}$ is the surface of the $i$-th colloid with outward pointing surface normal $\mathbf{n}_{i}$. Constantcharge boundary conditions are assumed for all $N$ colloid surfaces [10]. The PB-MD simulation algorithm is now described as follows [9]: (i) fix all $N$ colloids at their initial positions $\left\{\mathbf{R}_{i}\right\}^{0}$, (ii) solve the PB eq. (1) for this colloidal configuration, (iii) calculate the total force on each colloid by integrating the stress-tensor over a surface enclosing the respective particle, (iv) move all colloids to their new positions by an Euler MD time-step including a stochastic force modeling the heat bath. Repeat (ii - iv). The solution of the PB equation in step (ii) is computationally the most demanding part of the calculation since it is necessary to resolve the steep variation of $\phi$ near the colloid surfaces. To achieve this resolution with a reasonable number of gridpoints, $N$ spherical coordinate systems are constructed, one centered around each colloid, which overlap with a Cartesian system in the cubic simulation box. The solution is obtained by (iia) solving the $\mathrm{PB}$ equation in each of the spherical systems for fixed values at the outer edges, (iib) interpolating to find the boundary values in the Cartesian system, (iic) solving the PB equation in the Cartesian system, and (iid) interpolating back to find new boundary values at the edges of the spherical systems. These steps are iterated to convergence. There are a few studies [11, 12, 13, 8] where similar simulations have been performed, but they mainly concentrate on determining the structure of colloidal dispersions. The method used by Löwen et al. [11, 12] is based on a density functional approach and includes also the microion correlations. However, it has been shown in various studies [14, 15] that the microion correlations play only a negligible role in case of monovalent salt ions as considered in this paper. Groot [14] quantified the validity of the PB approach by comparing it to a cell model Monte-Carlo simulation and showed that the deviations are already tiny for a ratio $\lambda_{B} / a=0.03$. Since we are here considering an even smaller value $\lambda_{B} / a=0.012$, the $\mathrm{PB}$ approach is perfectly justified relying on Groot's work.

Using the simulation method just described, we have calculated the melting line in the phasediagram of charge-stabilized colloidal suspensions. To facilitate later comparison of our results with the classic phase-diagram of Yukawa systems calculated by Robbins, Kremer and Grest (RKG) [17], we followed their work and determined the phase-boundary by the Lindemann rule which states that a crystal begins to melt when the rms displacement in the solid phase is a fraction of $19 \%$ of $d_{\mathrm{m}}$. Taking the radius $a$ of the colloidal spheres as unit-length scale and having in mind an aqueous system at room temperature where $\lambda_{B}=7.2 \AA$ and colloid particles of size $a=60 \mathrm{~nm}$ as common in experiments we fix the ratio $\lambda_{B} / a=0.012$. The state space of our colloidal system then is three-dimensional, spanned by $\kappa a$ (salt concentration), $Z$ (colloidal charge) and $d_{\mathrm{m}} / a$ (colloid density), or, equivalently, the volume-fraction $\eta=4 \pi\left(a / d_{\mathrm{m}}\right)^{3} / 3$. A point $\vec{X}$ in state space is thus given by $\vec{X} \equiv(\kappa a, Z, \eta)$. Since a systematic exploration of the full 3D phase-diagram is too expensive computationally, we focus on a $2 \mathrm{D}$ cut at large volume fractions $\eta=0.03$ realized in experiments. The resulting phase-diagram in the $(Z, \kappa a)$-plane shown in Fig. (1. a) represents our main result. It describes the solid-liquid phase behavior 

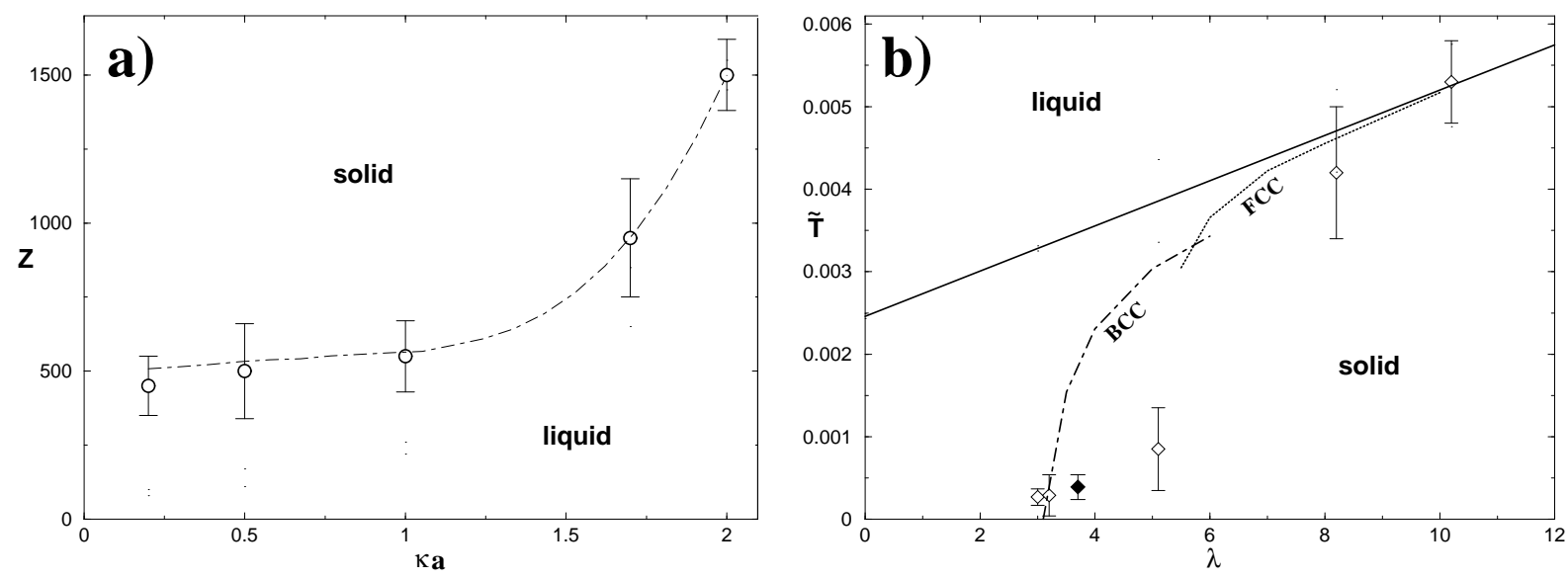

Figure 1: (a) Solid-liquid phase diagram of a charge-stabilized colloidal suspension, spanned by the colloidal charge $Z$ and the salt concentration $\kappa a$ for volume fraction $\eta=0.03$ and $\lambda_{B} / a=0.012$. The circles are points on the melting line obtained by applying the Lindemann criterium to PB-MD simulation data in a FCC crystal which fully include many-body interactions between the colloids. The line is a guide to the eyes. (b) The melting line of a pure Yukawa system obtained by Robbins et al. [17] (solid line), the melting line obtained assuming Yukawa interactions with a density-dependent cut-off after the first neighbor shell in a FCC configuration (dotted line) and in a BCC configuration (dot-dashed line), and the data from Fig. (1.a) transfered by using the effective force parameters as described in the text.

of colloidal suspensions with all many-body interactions among the colloids included. To our knowledge, it is the first such phase diagram calculated without assuming pairwise additive effective interactions. Its significance is best appreciated by contrasting it to the predictions based on the established description in terms of pairwise Yukawa potentials, which in turn requires to first introduce the concepts of charge renormalization and effective forces.

For a system of point-like Yukawa particles, interacting via $u(r)=U_{0} e^{-\lambda r / d_{\mathrm{m}}} /\left(r / d_{\mathrm{m}}\right)$, the state space is two-dimensional and spanned by $U_{0}$ and $\lambda$. In this space the melting line is a function $U_{0}^{M}(\lambda)$ that has been determined by RKG. More precisely, they introduced an effective temperature $\tilde{T}$ ( $k T$ in units of the Einstein phonon energy) which is related to $U_{0}$ by $\tilde{T}(\lambda)=$ $\left(2 \lambda^{2} \theta(\lambda) \beta U_{0}(\lambda) / 3\right)^{-1}$ where the function $\theta(\lambda)$ is given in Tab. I of 17]. They then determined the melting line as $\tilde{T}^{M}(\lambda)=0.00246+0.000274 \lambda$ which is plotted as the solid line in Fig. (11.b). For colloidal systems, we recall that the Yukawa pair-potential of DLVO theory reads

$$
\beta u(r)=\left[\frac{Z_{\mathrm{eff}} e^{\kappa_{\mathrm{eff}} a}}{1+\kappa_{\mathrm{eff}} a}\right]^{2} \lambda_{B} \frac{e^{-\kappa_{\mathrm{eff}} r}}{r}
$$

with the effective colloidal charge $Z_{\text {eff }}$ and the effective screening parameter $\kappa_{\text {eff }}$. These effective (or renormalized) quantities, introduced to capture effects arising from the non-linearity of the PB equation [18], are both (unknown) functions of $\vec{X}, \kappa_{\text {eff }} a=f_{1}(\vec{X})$ and $Z_{\text {eff }}=f_{2}(\vec{X})$. One way, among others, to approximately determine these functions is to use the PB cell model [16, 19. Identifying the parameters in eq. (2) with $U_{0}$ and $\lambda$ from the RKG calculation as 

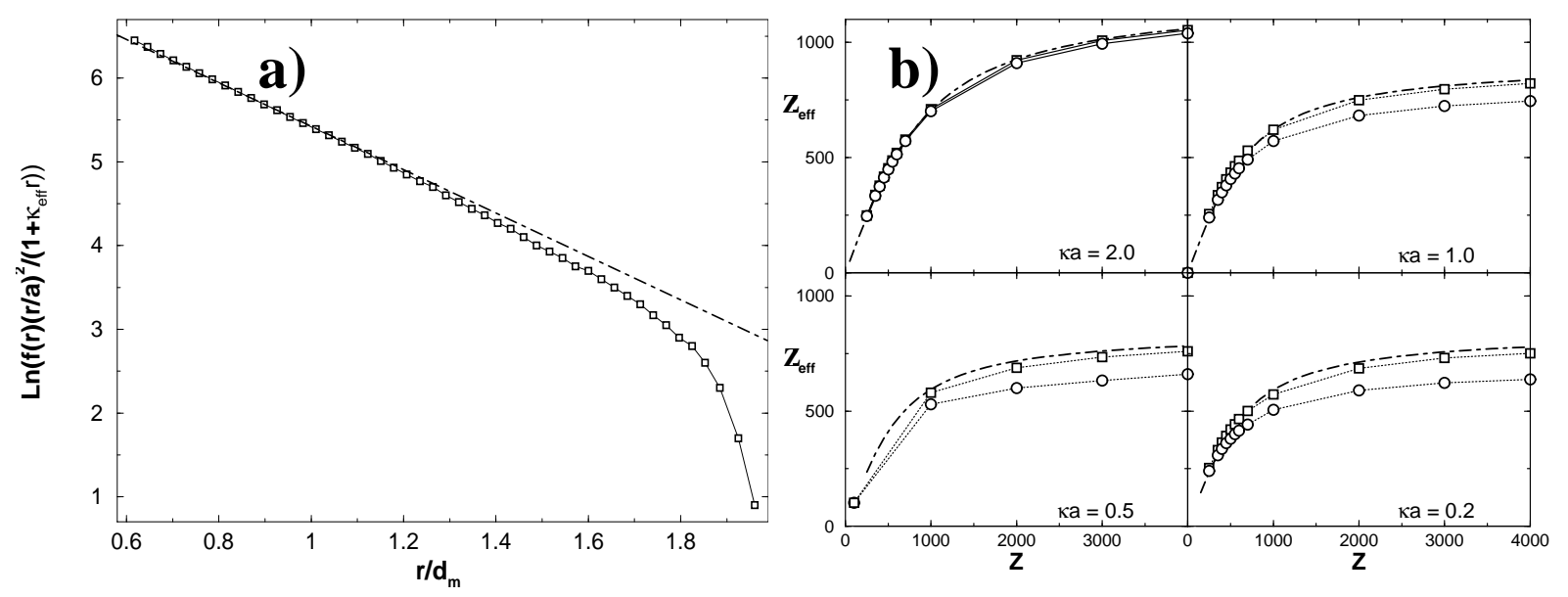

Figure 2: (a) Effective colloid-colloid pair-force as function of the distance between two colloids surrounded by 106 other colloids in a FCC configuration at $\eta=0.03, Z=1000, \kappa a=0.2$, $\lambda_{B} / a=0.012$. The dot-dashed line is the best fitting Yukawa interaction. (b) $Z_{\text {eff }}$ vs. $Z$, obtained from fitting Yukawa pair-forces to effective force curves in BCC (squares) and FCC (circles) configurations, for four salt concentrations as indicated and $\eta=0.03, \lambda_{B} / a=0.012$. The dot-dashed lines gives predictions of the PB cell model [16] for comparison.

$\beta U_{0}=\left(Z_{\text {eff }} e^{\kappa_{\text {eff }} a} /\left(1+\kappa_{\text {eff }} a\right)\right)^{2} \lambda_{B} / d_{\mathrm{m}}$ and $\lambda=\kappa_{\text {eff }} d_{\mathrm{m}}$, one can then use the RKG phase-diagram in Fig. (1.b) to estimate whether a colloidal system, represented by a state point $\vec{X}$, is solid or liquid. Note first that since the phase-behavior of a pure Yukawa system is determined just by $U_{0}$ and $\lambda$, the natural $3 \mathrm{D}$ state space of colloidal systems is reduced here to 2D. Secondly, it is clear that the data in Fig. (11.a) can be transfered to the phase-diagram of Fig. (11.b), only if the two functions $f_{1}(\vec{X})$ and $f_{2}(\vec{X})$ are provided. Then a data point at $(Z, \kappa a)$ in Fig. (1).a) can be transformed to $\left(Z_{\text {eff }}, \kappa_{\text {eff }} a\right)$ and subsequently plotted as $(\tilde{T}, \lambda)$ in Fig. (11.b). This requires two steps: (i) to calculate the effective pair-interaction (depending on the state $\vec{X}$ ), which includes the original many-body interactions and, in general, is not a Yukawa potential and then try (ii) to fit these pair-interactions to Yukawa potentials.

We proceed with the first task of finding an effective pair-interaction by performing step (i) to (iii) of the simulation algorithm described above for fixed colloidal configurations. The force $\vec{F}_{A B}$ exerted by a particle $\mathrm{A}$ on another particle $\mathrm{B}$ is obtained as the difference between the total force acting on particle $\mathrm{B}$ with particle A present, $\vec{F}_{B}^{1}$, and the total force acting on particle B, $\vec{F}_{B}^{0}$, after removing particle A while leaving all other particles at their positions [20]. Varying the position of particle A results in the effective force curve $\vec{F}_{A B}(\vec{r})=\vec{F}_{B}^{1}(\vec{r})-\vec{F}_{B}^{0}$. With this procedure the many-body interactions are folded into an effective pair interaction. If the true interactions in the system are pairwise additive, the resulting effective interaction is by construction identical to the true pairwise interaction potential, independent of the arrangement of the surrounding particles (all particles but A and B). This calculation has been carried out for FCC $(N=32$ and 108) and BCC $(N=54)$ configurations at different salt concentrations between $\kappa a=0.1$ and $\kappa a=2.0$, bare colloidal charges between $Z=10$ and $Z=4000$, and 
several volume fractions $\eta=0.01,0.02,0.03$. A typical effective force curve $\vec{f}(r)=a \beta \vec{F}_{A B}(r)$ in a low-salt system $(\kappa a=0.2)$ is presented in Fig. (2.a). Forces are multiplied by $(r / a)^{2} /\left(1+\kappa_{\mathrm{eff}} r\right)$ and plotted logarithmically so that the Yukawa interaction appears as a straight line with slope $-\kappa_{\text {eff }}$. Obviously, for small distances the effective forces are Yukawa-like. However, around the mean colloid-colloid distance $d_{\mathrm{m}}$ systematic deviations become visible which develop into a cutoff at $r>d_{\mathrm{m}}$. At the highest salt concentrations considered $(\kappa a=2.0)$, no such deviation occur in our calculation. A similar behavior has also been found in primitive model calculations [6].

As a second step, the values of the effective parameters $Z_{\text {eff }}$ and $\kappa_{\text {eff }}$ are obtained by fitting the force curve to the derivative of eq. (2) at $r \leq d_{\mathrm{m}}$, that is, in the range where the force is Yukawa-like. Performing the fit for all calculated force curves yields the dependence of the effective parameters on the state of the system, i.e., the two functions $f_{1}(\vec{X})$ and $f_{2}(\vec{X})$. The dependence of $Z_{\text {eff }}$ on $Z$ for different salt concentrations is shown in Fig. (2.b). The values obtained in FCC and BCC configuration are compared to the curves obtained from the PB cell model using the Alexander prescription [16, 19]. The predictions of the cell model agree with our results in the high-salt case $(\kappa a=2.0)$, but not under low salt conditions $(\kappa a=0.2)$. Moreover, we find agreement between the FCC and BCC results at $\kappa a=2.0$ but substantial differences at $\kappa a=0.2$. As noted above, such a configuration dependence $\left(f_{1,2}^{\mathrm{BCC}}(\vec{X}) \neq f_{1,2}^{\mathrm{FCC}}(\vec{X})\right)$ of the effective pair-interaction parameters is another fingerprint of the many-body interactions in the system next to the observed cut-off feature. A dependence of the pair-interaction on the colloidal configurations has also been found in [12]. In addition, attractive three-body forces have been explicitly shown to become effective in the low salt regime $(\kappa a<1)$ [21. The range of the effective interaction in the high salt calculation $(\kappa a=2.0)$, is about $1 / \kappa_{\text {eff }} \approx 0.5 a$, while for the low salt case $(\kappa a=0.2)$ it is roughly $2 a$. The mean distance $d_{\mathrm{m}}$ in both cases is about $5 a$. The onset of the configuration dependence thus coincides with the size of the double layer becoming comparable to $d_{\mathrm{m}}$ as pointed out in the introduction.

Having determined the functions $f_{1}(\vec{X})$ and $f_{2}(\vec{X})$ the data for the melting line in Fig. (1.a) can be transfered to Fig. (11.b), essentially by rescaling twice the $x$ - and y-axis of Fig. (1.a) $\left((Z, \kappa a) \rightarrow\left(Z_{\text {eff }}, \kappa_{\text {eff }} a\right) \rightarrow(\tilde{T}, \lambda)\right)$. Good agreement with RKG is obtained in our high-salt calculation $\kappa a=2.0$, corresponding to large values of $\lambda$, which is consistent with our finding that at high salt neither a cut-off behavior nor a configuration-dependence of the pair-potential could be observed. Obviously, in this salt regime the colloidal suspension can be represented by a Yukawa system quite well. However, reducing the amount of salt, i.e., decreasing $\lambda$, we observe pronounced deviations from the RKG line occurring in the low salt regime. Again, this matches with the behavior of the calculated effective force curves at low salt, showing a configuration dependence but also the cut-off feature. Both observations suggest that the difference between the RKG melting line and ours is due to many-body effects.

For practical applications it would of course be highly desirable to have a way of predicting the phase behavior of colloidal systems from a simple density-dependent pair-potential. The cutoff behavior observed in our effective force curves at low salt concentration and in the experiment 
in [5] suggests to use a truncated Yukawa potential

$$
u(r)= \begin{cases}U_{0} \frac{e^{-\lambda r / d_{\mathrm{m}}}}{\left(r / d_{\mathrm{m}}\right)} & r \leq r_{c} \\ 0 & r>0\end{cases}
$$

with a density-dependent cut-off $r_{c} \propto d_{\mathrm{m}}$ to include the macro-ion shielding effect [6]. With this model potential, we have carried out MD simulations and again determined the solidliquid phase-boundary by the Lindemann criterion, computing the rms displacement for various combinations of $U_{0}$ and $\lambda$ in FCC and BCC crystals. For $r_{c}=3.07 d_{\mathrm{m}}$, the cut-off used for numerical reasons in [17], the RKG melting line is reproduced. Upon decreasing the cut-off, systematic deviations from the RKG melting line are observed occurring first at small values of $\lambda$. The dotted line in Fig. (1. b) is the melting line obtained choosing $r_{c}=1.35 d_{\mathrm{m}}$ in a FCC configuration, while the dash-dotted line is the melting line in a BCC configuration choosing the cut-off radius $r_{c}=1.50 d_{\mathrm{m}}$. These two cut-off radii correspond to the first neighbor shell in the FCC and to the second neighbor shell in the BCC configuration so that the number of neighbors that are included in the interaction is comparable in both cases. By comparing these results to our full PB-MD simulation it is seen that the density-dependent cut-off provides a considerable improvement over the plain Yukawa potential. Since the cut-off in this model pair-interaction is related in a simple way to the macro-ion shielding effect this further corroborates our conclusions about the importance of many-body interactions for the solid-liquid phase behavior of colloidal suspensions.

Considering three-body forces for three colloids in an electrolyte, [21], one can show that the macroion shielding effect becomes less efficient with decreasing $a$. This implies that the observed difference between the RKG line and our line should become smaller with decreasing $\eta=4 \pi\left(a / d_{\mathrm{m}}\right)^{3} / 3$, which indeed we have observed in reference calculations at smaller volume fractions $(\eta=0.01$ and $\eta=0.005)$. This is, first of all, a reminder of the fact, already discussed above, that the phase-diagram of colloidal suspension depends on three quantities, while the Yukawa phase-diagram is just a $2 \mathrm{D}$ representation of it. Secondly, this is the reason why the existing experimental data in [22, 23] can not be used to validate our results, as these experiments have been performed at even smaller volume fractions $\left(10^{-3}\right.$ to $\left.10^{-4}\right)$. For such low volume fractions we do not expect noticeable many-body effects. We would therefore suggest to perform experiments similar to the ones in [22, 23] at higher volume fractions comparable to $\eta=0.03$ considered in our simulation.

A charge-stabilized colloidal suspension is often considered as a simple Yukawa liquid. This is not always correct. In conclusion, this paper shows that a Yukawa description of colloidal suspensions fails to predict the correct solid-liquid phase behavior under low-salt conditions where the range of the effective interaction is comparable to the mean distance and where many-body effects start playing a vital role. We have demonstrated this with the help of effective force calculations which have revealed a configuration dependence of the pair-interactions and deviations from Yukawa behavior, at $r \geq d_{m}$ and low salt. Model pair-potentials with density-dependent cut-offs have been shown to reproduce quite well the effects which many-body interactions have on the phase-behavior of colloidal suspension. We predict many body effects to increase with 
increasing colloid volume fraction.

We gratefully acknowledge useful and stimulating discussions with R. Klein, C. Bechinger, M. Brunner, Y. Chen, M. Brumen, D. Haložan and C Russ.

\section{References}

[1] Verwey E. J. W. and Overbeek J. T. G., Theory of the Stability of Lyophobic Colloids, Elsevier, New York, 1948

[2] Crocker J. C. and Grier D. G., Phys. Rev. Lett., 73, 1994, 352

[3] Vondermasse K., Bongers J., Mueller A., and Versmold H., Langmuir, 10, 1994, 1351

[4] Crocker J. C. and Grier D. G., Phys. Rev. Lett., 77, 1996, 1897

[5] Brunner M., Bechinger C., Strepp W., Lobaskin V., and von Grünberg H. H., Europhys. Lett., 58, 2002, 926

[6] Klein R., von Grünberg H. H., Bechinger C., Brunner M., and Lobaskin V., J. Phys.: Condens. Matter, 14, 2002, 7631

[7] Louis A. A., J. Phys. Cond. Matter, 2002, in press (cond-mat 0205110)

[8] Fushiki M., J. Chem. Phys., 97, 1992, 6700

[9] Dobnikar J., Rzehak. R. and von Grünberg H. H., preprint, 2002

[10] Integrating the microioinic density distribution over $G$ results in the total number of microions in the system, which allows to compute $n_{s}$, the concentration of salt in the system. The latter is to be distinguished from the reservoir salt concentration $c_{s}$ (due to the Donnan effect $n_{s} \leq c_{s}$ ).

[11] Löwen H., Hansen J. P., and Madden P. A., J. Chem. Phys., 98, 1993, 3275

[12] Löwen H. and Kramposthuber G., Europhys. Lett., 23, 1993, 673

[13] Tehver R., Ancilotto A., Toigo F., Koplik J., and Banavar J. R., Phys. Rev. E, 59, 1999, R1335

[14] Groot R. D., J. Chem. Phys., 95, 1991, 9191

[15] Carnie S. L., and Torrie G. M., Adv. Chem. Phys., 56, 1984, 142

[16] Alexander S., Chaikin P. M., Grant P., Morales G. J., Pincus P. and Hone D., J. Chem. Phys., 80, 1984, 5776

[17] Robbins M. O., Kremer K., and Grest G. S., J. Chem. Phys., 88, 1988, 3286

[18] Belloni L., Colloids Surfaces A, 140, 1998, 227 
[19] Bocquet L., Trizac E. and Aubouy M., J. Chem. Phys., in press, 2002

[20] L. Belloni, private communication.

[21] Russ C., van Roij R., Dijkstra M., and von Grünberg H. H., Phys. Rev. E, 66, 2002, 011402-1

[22] Monovouskas Y. and Gast A. P., J. Colloid Interface Sci.128, 1989, 533

[23] Liu J., Schöpe H. J. and Palberg T.,J. Chem. Phys.116, 2002, 5901 